\section{Biserial and point-biserial correlation with correction for nonoptimal dichotomies}

\author{
WILLIAM P. DUNLAP \\ Tulane University, New Orleans, Louisiana \\ and \\ EDWARD R. KEMERY \\ University of Baltimore, Baltimore, Maryland
}

In a recent paper on the cost of dichotomizing variables, Cohen (1983) addressed the impact of having unequal proportions in each category of a dichotomous variable. When a continuous bivariate normal variable is dichotomized at the mean of the underlying distribution (i.e., $p=q=.5$ ), the upper limit of the observed correlation will be equal to 0.798 times the population correlation. In instances in which a variable has been dichotomized at either $0.5,1.0$, or 1.5 standard deviations from the mean, the observed correlation will be reduced by a factor of $0.762,0.662$, and 0.519 , respectively. In other words, if a variable was dichotomized at 1.5 standard deviations from the mean (i.e., $p=.841$ and $q=.159$ ) and the underlying population product-moment correlation was 0.7 , the observed correlation would be .3633 (i.e., . $519 \times .7$ ).

Kemery, Dunlap, and Griffeth (in press) derived and empirically tested a procedure for correcting point-biserial correlations based on a particular $p / q$ split in the dichotomous variable, to estimate what that correlation would have been had an optimum $.5 / .5$ split occurred for the dichotomous variable. This procedure first involves calculating the biserial correlation, which is an estimate of the correlation between two continuous normal variables, assuming that the dichotomized variable was originally normal and had been arbitrarily dichotomized at the point $p$. The biserial $r$ can then be converted back to show what the point-biserial $r$ would have been with an optimal $.5 / .5$ split, or any other $p / q$ split.

Mathematical Development. Let $p$ equal the proportion in the first category and $q=1-p$ be the proportion in the second category of the dichotomized variable. When the second variable is continuous, the correlation coefficient is called the point-biserial correlation, $r_{p b}$, and the Pearson equation simplifies to

$$
r_{p b}=\left[\left(Y_{P}-Y_{q}\right)(p q)^{1 / 2}\right] / S_{Y},
$$

where $Y_{p}$ and $Y_{q}$ are the respective means of the continuous variable for the two categories of the binomial variable, and $S_{Y}$ is the standard deviation of the continuous variable. Because this equation contains the crossproduct term, $p q$, if the $Y$ means and standard deviation remain

\footnotetext{
Address correspondence to William P. Dunlap, Department of Psychology, Tulane University, New Orleans, LA 70118.
}

the same, the correlation will be maximal for $p=0.5$, and will be increasingly attenuated as a function of the disparity between $p$ and $q$. Because $(p q)^{1 / 2}$ is the standard deviation of the dichotomized variable, this is analogous to the problem of range restriction attenuating the correlation between continuous variables.

In order to correct $r_{p b}$ for a nonoptimum $p / q$ split, one must think of the dichotomous variable as having arisen from an arbitrary cut through an underlying continuous distribution. Take, for example, the problem of predicting employee turnover. If the tendency to quit or change jobs has an underlying continuous distribution, then reexamining a cohort after a given elapsed time period represents just such an arbitrary cut; if one had waited a longer period, more of the group would have quit. If it can further be assumed that this underlying distribution is normal, then it is possible to estimate the correlation that would have existed if the underlying distribution were directly measured and correlated. This estimated underlying correlation is called the biserial correlation, $r_{b}$, and is related to $r_{p b}$ by

$$
r_{b}=r_{p b}(p q)^{1 / 2 / h},
$$

where $h$ is the height of the standard normal distribution at the point where the cut was made, $p$ the area to the left, and $q$ the area to the right. To convert from $r_{b}$ back to $r_{p b}$, the conversion factor is obviously $h /(p q)^{1 / 2}$. Therefore, to estimate what $r_{p b}$ would have been had the cutting point been at 0.5 , one first converts to $r_{b}$, then backconverts to $r_{p b}$ with $p=q=0.5$. The resulting conversion factor is

$$
.7978(p q)^{1 / 2 / h}
$$

Using $h /(p q)^{1 / 2}$, one can convert the biserial $r$ back to the point-biserial $r$ that would have resulted from any other $p / q$ split. Because these computations use population equations, the program may occasionally produce bizarre outcomes, such as $r_{b}$ or corrected $r_{p b}$ greater than one. This should happen only rarely if reasonable sample sizes are used. Also, the equations assume bivariate normality and linearity of regression; thus they can be expected to be accurate only when the standard correlation assumptions obtain.

The Program. Three forms of initial input are permitted: a previously computed point-biserial $r$, together with respective sample sizes, can be entered directly; the pointbiserial correlation can be computed from raw data; and the point-biserial correlation can be computed from group means, standard deviations, and sample sizes. The program outputs the point-biserial correlation, the corresponding biserial correlation, and the point-biserial correlation corrected to a $.5 / .5$ split. The program then inquires whether the user wishes to correct $r_{p b}$ for other possible $p / q$ splits in the dichotomous variable. The only 
nontrivial part of these latter computations is finding values for $h$, the ordinate of the normal distribution at the point $p$. To find $h, p$ must first be converted to the corresponding $z$ score, which is done by the inverse normal algorithm of Odeh and Evans (1974). Entering this $z$ value into the equation for the normal curve gives $h$.

Requirements. The program (see the Appendix) is written in FORTRAN and runs on an IBM-PC, and should run on any computer with a FORTRAN IV or later compiler. All computations are done in single precision, which produces approximate five- to six-place accuracy. Modifying the program to run on other computers should require modification only in the specification of input and output units, which at present are the keyboard and screen of the PC. All input is free format; therefore, when more than one value is requested, multiple values should be separated by commas.

Availability. A listing of the program can be obtained at no charge from William P. Dunlap. To receive the compiler IBM-PC version together with the source code, please send a double-sided, double-density, soft-sectored, $51 / 4$-in. floppy disk with your request.

\section{REFERENCES}

Cohen, J. (1983). The cost of dichotomization. Applied Psychological Measurement, 7, 249-253.

ODEh, R. E., Evans, J. O. (1974). The percentage points of the normal distribution. Applied Statistics, 23, 96-97.

Kemery, E. R., Dunlap, W. P., \& Griffeth, R. W. (in press). Correction for variance restriction in point-biserial correlations. Joumal of Applied Psychology.

\section{APPENDIX}

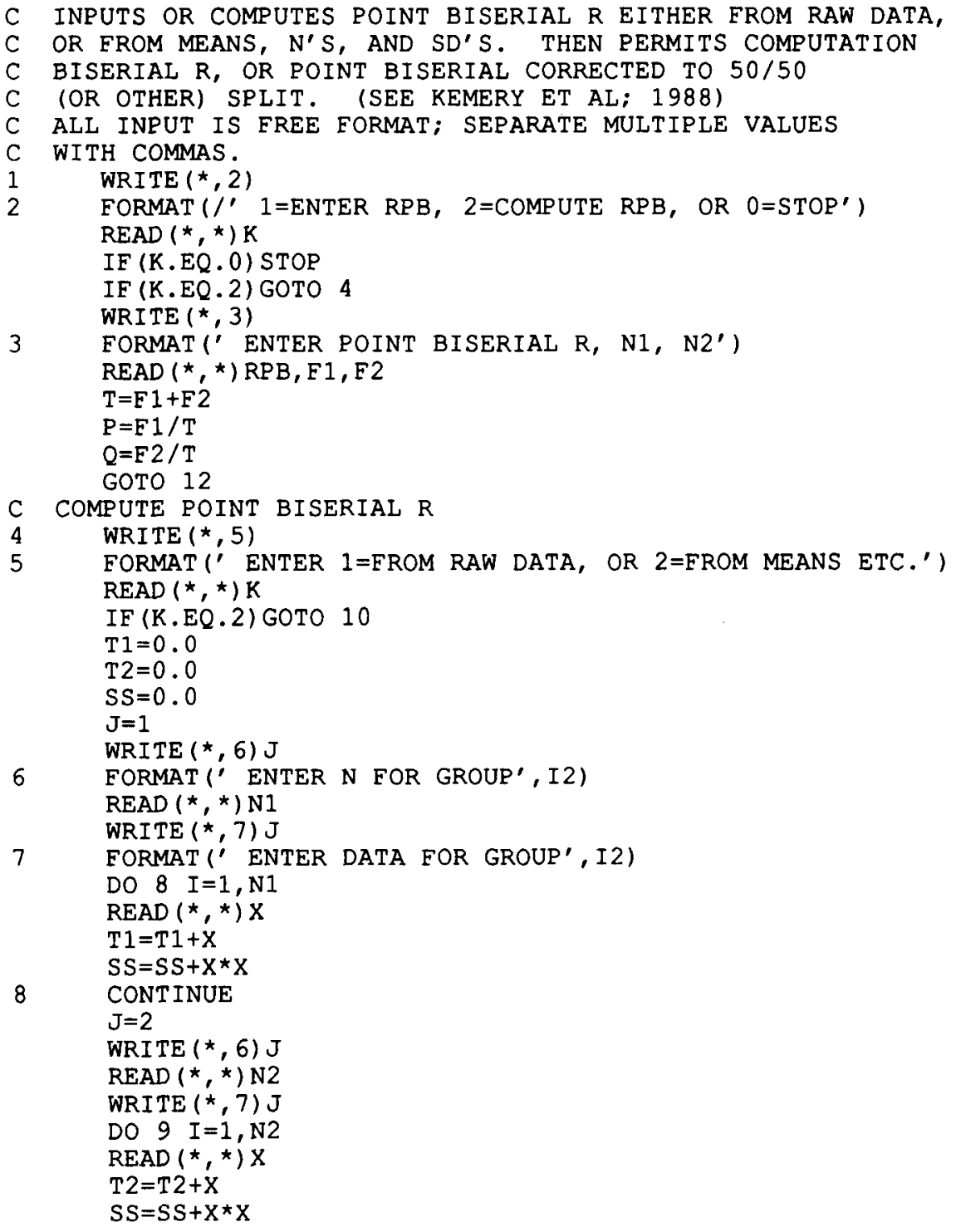


9

CONTINUE

$\mathrm{E} 1=\mathrm{N} 1$

$\mathrm{F} 2=\mathrm{N} 2$

$\mathrm{T}=\mathrm{N} 1+\mathrm{N} 2$

$\mathrm{P}=\mathrm{F} 1 / \mathrm{T}$

$\mathrm{Q}=\mathrm{F} 2 / \mathrm{T}$

$\mathrm{SS}=\mathrm{SS}-(\mathrm{T} 1+\mathrm{T} 2) * \star 2 / \mathrm{T}$

$\mathrm{XB} 1=\mathrm{T} 1 / \mathrm{F} 1$

$\mathrm{XB} 2=\mathrm{T} 2 / \mathrm{F} 2$

$\mathrm{RPB}=(\mathrm{XB} 2-\mathrm{XB} 1) \star \mathrm{SQRT}(\mathrm{P} * \mathrm{Q}) / \mathrm{SQRT}(\mathrm{SS} / \mathrm{T})$

GOTO 12

$10 \mathrm{~J}=1$

WRITE (*,11) J

11 FORMAT (' ENTER MEAN, S, N FOR GROUP', I2)

$\operatorname{READ}(*, *) \mathrm{XB} 1, \mathrm{~S} 1, \mathrm{~F} 1$

$\mathrm{J}=2$

WRITE $(*, 11) \mathrm{J}$

$\operatorname{READ}(\star, \star) X B 2, \mathrm{~S} 2, \mathrm{~F} 2$

$\mathrm{T}=\mathrm{F} 1+\mathrm{F} 2$

$\mathrm{P}=\mathrm{F} 1 / \mathrm{T}$

$\mathrm{Q}=\mathrm{F} 2 / \mathrm{T}$

$\mathrm{SS} 1=\mathrm{S} 1 * \mathrm{~S} 1 *(\mathrm{~F} 1-1.0)+\mathrm{F} 1 * \mathrm{XB} 1 * \mathrm{XB} 1$

$\mathrm{SS} 2=\mathrm{S} 2 * \mathrm{~S} 2 *(\mathrm{~F} 2-1.0)+\mathrm{F} 2 * X \mathrm{~B} 2 * \times \mathrm{B} 2$

$\mathrm{SS}=\mathrm{SS} 1+\mathrm{SS} 2-\left(\mathrm{XB} 1{ }^{\star} \mathrm{F} 1+\mathrm{XB} 2 \star \mathrm{F} 2\right) \star \star 2 / \mathrm{T}$

12 WRITE $(*, 13)$ RPB $, P, Q$

$\mathrm{RPB}=(\mathrm{XB} 2-\mathrm{XB} 1) * \mathrm{SQRT}(\mathrm{P} * \mathrm{Q}) / \mathrm{SQRT}(\mathrm{SS} / \mathrm{T})$

13 FORMAT $(/$, POINT BISERIAL CORRELATION $=\prime$, F 10.6 ,

* $, \quad P=\prime, F 10.6, ' \quad Q=\prime, F 10.6$ )

$\mathrm{Z}=\mathrm{Z} \operatorname{INV}(\mathrm{P})$

$\mathrm{H}=.3989423^{\star} \operatorname{EXP}(-\mathrm{Z} \star \mathrm{Z} / 2)$

$\mathrm{RB}=\mathrm{RPB} * \mathrm{SQRT}(\mathrm{P} * \mathrm{Q}) / \mathrm{H}$

$\mathrm{RFF}=\mathrm{RB} * .7978846$

14

WRITE (* 14) RB, RFF

FORMAT $(/, \quad$ DERIVED COEFFICIENTS $/ \prime$
$*$ F $10.6 /, \quad \operatorname{RPB}(\mathrm{P}=.5, \mathrm{Q}=.5)=\prime, \mathrm{F} 10.6)$

15 WRITE $(*, 16)$

16 FORMAT ( $/$ 'ENTER $1=$ CORRECT FOR ANOTHER SPLIT, $0=$ RETURN $\left.{ }^{\prime}\right)$

$\operatorname{READ}(*, *) \mathrm{K}$

IF (K.EQ.0) GOTO 1

WRITE $(*, 17)$

FORMAT (/' ENTER P, Q FOR NEW SPLIT')

$\operatorname{READ}\left({ }^{*},{ }^{\star}\right) \mathrm{P}, \mathrm{Q}$

$\mathrm{T}=\mathrm{P}+\mathrm{Q}$

$\mathrm{P}=\mathrm{P} / \mathrm{T}$

$\mathrm{Q}=\mathrm{Q} / \mathrm{T}$

$\mathrm{Z}=\mathrm{ZINV}(\mathrm{P})$

$\mathrm{H}=.3989423^{\star} \operatorname{EXP}(-Z \star Z / 2)$

$\mathrm{RNS}=\mathrm{RB} * \mathrm{H} / \mathrm{SQRT}(\mathrm{P} * \mathrm{Q})$

WRITE (*,13) RNS, P,Q

GOTO 15

END

C

FUNCTION ZINV(P)

C $P=P R O B(Z>Z S C O R E)$. ODEH \& EVANS, 1974, APPLIED STAT, 23, 96-97.

DATA P0, P1,P2,P3/-.3222324,-1.0,-.3422421,-.2042312E-1/

DATA P $4, Q 0, Q 1 /-.4536422 \mathrm{E}-4, .9934846 \mathrm{E}-1, .5885816 /$

DATA $Q 2, Q 3, Q 4 / .5311035, .1035378, .3856070 \mathrm{E}-2 /$

$\mathrm{PS}=\mathrm{P}$

IF (P.GT . 0.5) PS $=1 .-\mathrm{P}$

$\mathrm{Y}=\operatorname{SQRT}(-2 . \star \mathrm{ALOG}(\mathrm{PS}))$

$\mathrm{ZINV}=\mathrm{Y}+((((\mathrm{P} 4 \star \mathrm{Y}+\mathrm{P} 3) \star Y+P 2) \star Y+P 1) \star Y+P 0) /((((Q 4 * Y+Q 3) \star Y+Q 2) \star Y+Q 1)$

$* \star \mathrm{Y}+\mathrm{Q})$

IF (P.GT.0.5) ZINV $=-Z I N V$

RETURN

END 